\title{
Attosecond nuclear dynamics in the ammonia cation: relation between high-harmonic and photoelectron spectroscopies
}

\author{
Journal Article \\ Author(s): \\ Kraus, Peter M.; Wörner, Hans Jakob \\ Publication date: \\ 2013-05-10 \\ Permanent link: \\ https://doi.org/10.3929/ethz-a-010779880 \\ Rights / license: \\ In Copyright - Non-Commercial Use Permitted \\ Originally published in: \\ ChemPhysChem 14(7), https://doi.org/10.1002/cphc.201201022
}




\title{
Attosecond nuclear dynamics in the ammonia cation: relation between high-harmonic and photoelectron spectroscopies
}

\author{
P. M. Kraus* $\quad$ H. J. Wörner*†
}

February 19, 2013

\section{Abstract}

We report measurements of the umbrella motion in the ammonia cation on the attosecond time scale. The motion is prepared by strong-field ionization and probed by photorecombination through the process of high-harmonic generation. Performing such measurements at multiple wavelengths $(0.8,1.44,1.8$ $\mu \mathrm{m}$ ) enables us to follow the nuclear dynamics over a broad temporal range (0.8-3.8 fs). The intensity of the driving field is found to have a significant impact on the observed dynamics through the vibrational-state dependence of the strong-field ionization rates. We derive a general model that includes these effects and establishes a new link between high-harmonic spectroscopy and classical photoelectron spectroscopy. Our model reproduces the observed dynamics and their dependence on the intensity of the driving field. Moreover, the model predicts much richer nuclear dynamics on the few-fs timescale than most previous theories. The newly predicted features are shown to reflect the quantized vibronic level structure of the molecular cation.

\section{Introduction}

The non-perturbative interaction between an intense laser field and a molecule enables the measurement of processes on timescales below the duration of an optical cycle. The laser-induced recollision between a molecular cation and an electron indeed provides the basis for several approaches to measuring attosecond intramolecular dynamics. Exploiting the correlation between the nuclear

${ }^{*}$ Laboratorium für Physikalische Chemie, ETH Zürich, Wolfgang-Pauli-Strasse 10, 8093 Zürich, Switzerland

†woerner@ethz.ch 
wave packet created in the molecular cation by strong-field ionization and the electronic continuum wave packet has enabled the measurement of nuclear dynamics on a subfemtosecond time scale $[1,2]$. The same concept also applies to high-harmonic spectroscopy (HHS) with the additional benefit of a unique relationship between the emitted photon energy and the time elapsed between ionization and recombination. The overlap integral of the neutral and ionic nuclear wave functions is thus mapped onto the intensity distribution of the emitted high-harmonic spectrum [3]. The squared ratio of nuclear autocorrelation functions for two isotopologues can thus be obtained from the ratio of their high-harmonic spectra. This principle enables the observation of nuclear dynamics in the molecular cation with a temporal resolution on the order of 100 as, as experimentally demonstrated in $\mathrm{D}_{2} / \mathrm{H}_{2}$ and $\mathrm{CD}_{4} / \mathrm{CH}_{4}$ [4]. The measurements on molecular hydrogen have been further investigated in terms of two-center interference [5] and extended to $1.3 \mu \mathrm{m}$ [6]. The nuclear dynamics were also investigated in mixtures of $\mathrm{D}_{2}$ and $\mathrm{H}_{2}$ in order to access phase differences of the high harmonics emitted in both species [7]. Using the reconstruction of attosecond beating by interference of two photon transitions (RABITT) technique, the relative spectral phases of $\mathrm{D}_{2}$ and $\mathrm{H}_{2}$ were investigated in [8]. Moreover, the technique was used to investigate strong-field ionization to multiple electronic states in the water cation [9]. Other studies investigated the influence of the nuclear dynamics on the production of attosecond pulses $[10,11]$. Theoretical work on polyatomic molecules has so far relied on the harmonic oscillator approximation, investigating the calculation of nuclear autocorrelation functions from $a b$ initio data [12] and the use of vibrational normal modes in modeling the observed dynamics of the methane cation [13].

Independently of these studies, the role of nuclear motion in strong-field ionization of $\mathrm{H}_{2}$ has been studied in detail. Franck-Condon factors alone have been found to be insufficient for describing the vibrational-state dependence of strong-field ionization rates, as shown e.g. in Refs. [14, 15]. It was recognized that the rate of strong-field ionization to specific vibrational states of the cation also needs to be weighted according to their different ionization energies [16]. More detailed calculations were carried out and found to reproduce the measured populations of the vibrational eigenstates of $\mathrm{H}_{2}^{+}$following strong-field ionization of $\mathrm{H}_{2}$ [17].

In this article, we investigate the relation between attosecond nuclear dynamics probed through the process of high-harmonic generation and classical photoelectron spectroscopy. As an illustration, we study the umbrella motion of the ammonia cation over a broad range of near-infrared (NIR) wavelengths and intensities of the generating laser pulse. The NIR wavelengths extending from 0.8 to $1.8 \mu \mathrm{m}$ provide access to longer timescales of the launched vibrational wave packet than previous results. Our experimental data reveal that the slope of the extracted nuclear autocorrelation function is smaller than expected from pure Franck-Condon factors. We show that this observation is explained by the vibrational-state dependence of the strong-field ionization rates, which reduces the energy bandwidth of the launched nuclear wave packet thus decelerating the induced nuclear dynamics. Our study of the intensity dependence of the 
extracted autocorrelation function shows in addition that the speed of the vibrational dynamics can be controlled through the intensity of the ionizing laser field.

We demonstrate these effects by deriving an accurate theoretical model that we validate through comparison with our measurements. Our model relies on an expansion of the wave packet describing the molecular cation into its vibronic eigenstates. We use experimental photoelectron spectra to determine the required Franck-Condon factors and thereby establish a new link between classical photoelectron spectroscopy and HHS. In addition, our model enables us to incorporate the vibrational-state dependence of strong-field ionization rates and to predict the manifestations of quantized vibronic structure in high-harmonic spectra. Our approach makes use of the Wiener-Khinchin theorem developed in stochastic data processing and later used to relate time- and frequency-domain spectroscopies [18]. Our model thus overcomes two stringent approximations used in previous models for polyatomic molecules, i.e. the assumed geometryindependence of the ionization rates and the harmonic oscillator approximation. Our model predicts small oscillations of the autocorrelation function after its initial decay that result from the vibrationally-resolved structure of the photoelectron spectrum. We expect that these effects will become experimentally observable as modulations in the spectral ratios for sufficiently long transit times beyond the data presented here.

\section{Theoretical model}

We first introduce a general theoretical model and then use it below to explain the experimental observations. As proposed by Lein [3], the envelope of the high-harmonic spectrum of a molecule undergoing ultrafast nuclear dynamics triggered by ionization is given by

$$
S^{\mathrm{HHG}}(\Omega) \propto|C(\tau(\Omega))|^{2} \cdot S_{\mathrm{fix}}^{\mathrm{HHG}}(\Omega),
$$

where $S_{\text {fix }}^{\mathrm{HHG}}(\Omega)$ is the high-harmonic spectral envelope obtained for fixed nuclei. $C(\tau)$ is the nuclear autocorrelation function given by the overlap integral of the nuclear wave packet prepared in the cation $\chi^{(+)}(R, \tau)$ with the ground state nuclear wave function $\chi^{(0)}(R, 0)=\phi_{0}^{(0)}(R)$ of the neutral molecule. For short electron trajectories, a unique relationship $\tau(\Omega)$ exists between the emitted frequency $\Omega$ and the transit time of the electron $\tau$ [3]. $R$ collectively represents the internal vibrational degrees of freedom of the molecule. This formulation neglects the coupling of electronic and nuclear degrees of freedom which is small in the electronic ground state of the ammonia cation. It also neglects the variation of the photorecombination matrix elements with nuclear geometry which is a good approximation in the absence of structural minima [5]. Finally our formulation neglects dynamics induced in the neutral molecule (e.g. through Lochfrass [19]). The latter is a valid approximation for measurements carried out at low intensities, such as those reported here. The nuclear wave packet 
of the cation can be written as a sum over the accessed vibronic eigenstates $\phi_{n}^{(+)}(R)$ of the cation, such that the autocorrelation function reads

$$
C(\tau)=\left\langle\chi^{(0)}(R, 0) \mid \chi^{(+)}(R, \tau)\right\rangle=\sum_{n} c_{n}\left\langle\phi_{0}^{(0)}(R) \mid \phi_{n}^{(+)}(R)\right\rangle e^{-i E_{n} \tau / \hbar},
$$

where $E_{n}$ is the energy of the $n^{\text {th }}$ vibronic eigenstate. Hence, the autocorrelation function can be related to the Franck-Condon factors $S_{n}^{F C}=\left|\left\langle\phi_{0}^{(0)}(R) \mid \phi_{n}^{(+)}(R)\right\rangle\right|^{2}$ through a Fourier transformation. The squared magnitudes of the expansion coefficients $c_{n}$ represent the populations of the eigenstates prepared by strong-field ionization. As observed and theoretically derived in [17], the populations can be expressed as products of Franck-Condon factors and the well-known ionizationenergy dependence of strong-field ionization, i.e. $c_{n}=\left\langle\psi_{n}^{(f)}(r, R)|\hat{V}| \psi_{n}^{(i)}(r, R)\right\rangle$, where $\psi_{n}^{(i)}(r, R)$ is the total wave function of the neutral molecule and $\psi_{n}^{(f)}(r, R)$ is the total wave function describing the ionized molecule and the continuum electron wave packet. $\hat{V}$ is the operator describing strong-field ionization. The transition moment becomes separable if the $R$-dependence of $\hat{V}$ is neglected, which corresponds to neglecting the variation of the molecular ionization potential with $R$. This assumption is justified when tunnel ionization is limited to a narrow range of nuclear coordinates, where the vibrational wave functions of the neutral and cationic state have maximal overlap. Thus, we obtain $\left|c_{n}\right|^{2}=S_{n}^{F C} \cdot r_{i o n}$, where the ionization rate can be approximated by $r_{\text {ion }}\left(I_{p}\right) \propto e^{-\frac{2}{3} \frac{\left(2 I_{p}\right)^{3 / 2}}{E}}[20]$, where $I_{p}$ is the ionization potential of the investigated system and $E$ is the laser electric field strength at the time of ionization.

We have thus shown how the nuclear autocorrelation function $C(\tau)$ can be determined from the vibronic energy level structure of the cation and the Franck-Condon factors. These quantities can be calculated by determining the Born-Oppenheimer potential energy surfaces of the relevant neutral and ionic electronic states followed by a solution of the nuclear Schrödinger equation. Since accurate calculations of this kind are challenging for polyatomic molecules, we propose instead to extract the required information from experimental photoelectron spectra. The intensity distribution of vibrationally resolved photoelectron spectra indeed closely reflects the Franck-Condon factors when vibronic coupling in the cation can be neglected. In our approach, the nuclear autocorrelation function relevant to high-harmonic generation can then be conveniently determined as the inverse Fourier transform of the photoelectron band corresponding to ionization from the ground state of the neutral molecule to the electronic ground state of the cation, multiplied with the strong-field ionization amplitude, i.e. $C(\tau)=\mathscr{F}^{-1}\left[I_{\mathrm{HOMO}}^{\mathrm{PES}} \cdot \sqrt{r_{\text {ion }}}\right]$. This relation establishes a simple formal link between classical photoelectron spectroscopy and the nuclear motion probed by high-harmonic generation. The only implicit approximation that we have made is that the Born-Oppenheimer potential energy surfaces of the ground electronic states of the neutral and ionic species are not affected by the laser field.

We implement our model by taking experimental photoelectron spectra of $\mathrm{NH}_{3}$ and $\mathrm{ND}_{3}$ from Ref. [21]. The positions and intensities of the vibronic 
transitions in the photoelectron spectra of the electronic ground state of $\mathrm{NH}_{3}^{+}$ (Fig. 2a) and $\mathrm{ND}_{3}^{+}$(not shown) were used in a least-squares fitting procedure employing an asymmetric Gaussian profile [22] to obtain the spectral envelopes of the photoelectron band. The width of the spectral envelope determines the energy bandwidth of the launched nuclear wave packet and thus its early time evolution. In Fig. 2b the squared autocorrelation functions calculated from the spectral envelopes of $\mathrm{NH}_{3}$ and $\mathrm{ND}_{3}$ are shown together with their ratio. In similarity to previous computations of the autocorrelation function ratio for $\mathrm{D}_{2} / \mathrm{H}_{2}$ and $\mathrm{CD}_{4} / \mathrm{CH}_{4}[3,4,13]$ a monotonic rise of the ratio is obtained.

Taking the vibrational structure of the spectrum into account as shown in Fig. 2c (the spectral resolution corresponds to the literature data in [21], but our analysis of the dynamics on the few-fs time-scale was tested to be insensitive to the linewidth of the vibronic structure), we observe that the autocorrelation function shows an intensity recurrence around $31.7 \mathrm{fs}$. This duration corresponds to one vibrational period of the umbrella motion of $\mathrm{NH}_{3}^{+}$and is determined by the energy spacing of the transitions observed in the photoelectron spectrum. It is thus a physically necessary result that shows the validity of the approach. The ratio of the squared autocorrelation functions of $\mathrm{ND}_{3}$ and $\mathrm{NH}_{3}$ in Fig. 2d initially shows the same behavior as in Fig. 2b, but a pronounced maximum is found at $4.8 \mathrm{fs}$. The reason for the occurrence of this maximum is that - as in electronic circuits - a sharp truncation of a signal in the frequency domain leads to oscillations in the time domain. A vibrationally-resolved photoelectron spectrum possesses such truncations in the form of its first and last vibronic bands. The resulting oscillations in the autocorrelation function of the investigated molecule and its deuterated counterpart will be displaced in time, causing the complex structure of the autocorrelation function ratio shown in Fig. 2d. In the present case, a local maximum of the ratio is predicted at $4.8 \mathrm{fs}$ that results from a maximal contrast between the autocorrelation functions of $\mathrm{ND}_{3}$ and $\mathrm{NH}_{3}$.

Taking into account the weighting of the vibrational states by their strongfield ionization rates as described above, the preparation of energetically higherlying vibrational states becomes less likely causing the corresponding transition strengths to decrease as shown in Fig. 2e for an intensity of $5 \cdot 10^{13} \mathrm{~W} / \mathrm{cm}^{2}$. The ratio of the autocorrelation functions still shows a maximum as shown in Fig. 2f, but with a much smaller amplitude than when the strong-field ionization correction is neglected as shown in Fig. 2d. This result is explained by the ionization-potential dependence of the strong-field ionization rate $r_{\text {ion }}$ that suppresses ionization to higher-lying vibronic states in the same way for the protonated and deuterated species. Therefore, the autocorrelation functions become more similar, damping the oscillations after the initial decay of the autocorrelation function and reducing the amplitude of the first local maximum in the ratio. An additional effect of the strong-field ionization weighting is that the induced nuclear dynamics become slower in general due to the energetic narrowing of the launched nuclear wave packet. In the following, we validate our model by comparing it with experimental data. 


\section{Experiment}

We generate high-harmonic radiation by focusing $(f=40 \mathrm{~cm}$ or $f=50 \mathrm{~cm})$ either the output of a Titanium:Sapphire (Ti:Sa) regeneratively amplified laser system $(800 \mathrm{~nm}, 25 \mathrm{fs})$ or the output of a high-energy optical parametric amplifier (TOPAS-HE, Light Conversion), which provides laser pulses with central wavelength tunable from $1.2 \mu \mathrm{m}$ to $2.4 \mu \mathrm{m}$, into a thin supersonic gas jet, generated by expansion of the gas samples through a 150-250 $\mu \mathrm{m}$ orifice. The contribution of the short electron trajectories is isolated by placing the gas jet 2-3 $\mathrm{mm}$ behind the position of the laser focus. The laser pulse durations from the TOPAS-HE amount to 40 - 50 fs measured with a home-built secondharmonic generation FROG (frequency-resolved optical gating) setup. The generated harmonics are spectrally dispersed by a concave XUV grating and imaged by a phosphor-backed double-stack microchannel-plate (MCP) detector, which is read out by a charge-coupled device (CCD) camera. $\mathrm{NH}_{3}$ (purity $\geq 99.98 \%$ ) and $\mathrm{ND}_{3}$ (isotopic purity $98 \%$ ) were purchased from Linde. The samples were seeded in Helium (1:3 to 1:9) with a backing pressure of 3-4 bars to reduce clustering, which was minimized by optimizing the contrast of the rotational revivals following impulsive alignment with an intense laser pulse. The rotational wave packet dynamics induced by a non-resonant $0.8 \mu \mathrm{m}$ laser pulse is probed by monitoring the intensity of high harmonics generated by a $1.3 \mu \mathrm{m}$ probe pulse as shown in Fig. 3. The high-harmonic intensity is found to maximize when the $\mathrm{C}_{3}$ axis of the $\mathrm{NH}_{3}$ molecule is aligned parallel to the polarization of the probe pulse and to minimize when the $\mathrm{C}_{3}$ axis is perpendicular. This observation is consistent with the nodal structure of the highest occupied molecular orbital of $\mathrm{NH}_{3}$ displayed as insets in Fig. 3. The timing of the rotational revivals and their high contrast shows that our experiments dominantly probe isolated molecules. Uncertainties in the gas density arise from the reproducibility of the pulsed nozzle operation, the laser power variations $(3 \%$ r.m.s. for the Ti:Sa amplifier output and $6 \%$ r.m.s. for the OPA output) and the accuracy of the power attenuation. Since we are interested in the slope of the ratios rather than in their absolute values, we have normalized them with a constant factor to obtain a spectral ratio of 1 for an extrapolated transit time of 0 fs.

\section{Results and Discussion}

We recorded high-harmonic spectra using laser pulses with central wavelengths of $0.8,1.44$ and $1.8 \mu \mathrm{m}$ and different intensities in order to access consecutive overlapping temporal ranges of the nuclear wave packet dynamics. We use the unique energy-to-time mapping within the classical model for the short electron trajectories $[23,4]$ to convert the energy scale of our recorded spectra into a time scale. High-harmonic spectra of $\mathrm{NH}_{3}$ and $\mathrm{ND}_{3}$ recorded at $0.8 \mu \mathrm{m}$ are shown in Fig. 4a. The peak laser intensity was estimated to be $1.5 \cdot 10^{14} \mathrm{~W} / \mathrm{cm}^{2}$ from the cutoff energy. The spectral ratio was calculated by integrating each observed harmonic separately. The result is shown in Fig. 4b with the hori- 
zontal axis converted into the transit time of the electron between ionization and recombination. At this wavelength, we access a temporal range of 0.7 $1.6 \mathrm{fs}$. The ratios predicted by our theoretical model are shown in the same panel. We present three different predictions of the autocorrelation-function ratios by Fourier transforming i) the vibrationally-resolved photoelectron spectrum (dashed red line), ii) the spectrum weighted with the ionization-potentialdependent strong-field ionization rates at the experimental intensity (full black line) or iii) by simply Fourier-transforming the spectral envelope (dash-dotted line). None of these three models has been discussed in the literature before. In all three cases similar increasing functions are obtained, which match the experimental data obtained at $0.8 \mu \mathrm{m}$ reasonably well. In this data set, it is hard to draw a clear conclusion about which approach best describes the observed dynamics. Therefore, neither the effect of the quantization of the vibronic structure nor the effect of the ionization rate is evident from the results obtained at $0.8 \mu \mathrm{m}$. We therefore turned to longer wavelengths and lower intensities to demonstrate the importance of these effects. A spectrum recorded with a central wavelength of $1.44 \mu \mathrm{m}$ and an intensity of $6.5 \cdot 10^{13} \mathrm{~W} / \mathrm{cm}^{2}$ is shown in Fig. 4c. The corresponding ratio and the model predictions are shown in Fig. 4d. In this case, the experimental ratio only agrees with the complete theoretical model (full black line), whereas the other predictions clearly deviate from the experimental result. To further validate our theoretical model, we recorded high-harmonic spectra at $1.8 \mu \mathrm{m}$, accessing transit times from $1.7-3.5 \mathrm{fs}$ of the launched nuclear wave packet and varied the intensities to demonstrate the influence of strong-field ionization rates on the population of the vibrational eigenstates (Fig. 4e and $\mathrm{f}$ ). In these measurements, the slope of the experimental ratios is smaller than in the measurements recorded at higher intensities because the populations of the energetically higher-lying vibrational states are suppressed by smaller strong-field ionization rates. Thus the temporal evolutions of the launched nuclear wave packets of the protonated and deuterated species become more similar consequently flattening the spectral ratio. Both simplified models, i.e. the one using only the envelope of the photoelectron band (green dash-dotted line) and the one neglecting the influence of the strong-field ionization rate for the different vibronic levels (red dashed line) strongly deviate from the experimentally observed dynamics. Only the full theoretical model provides an adequate description of the observed nuclear dynamics. Remaining discrepancies between experiment and theory might originate from the effect of the laser field on the potential energy surfaces or dynamics induced in the neutral molecule which have both been neglected in our model. The significant effect of the ionization rate on the nature of the prepared vibrational motion has thus been clearly demonstrated by comparing our experimental and theoretical results. The effect is most significant when the intensity of the generating field is low, such that only the lowest vibrational states are populated, and the wavelength is long, such that a large temporal range of the vibrational dynamics is accessed. We propose that the intensity control of the launched nuclear dynamics by strong-field ionization is a general concept. More explicitly, lowering the intensity of the driving field leads to a narrowing of the distribution 
of populated vibrational eigenstates, thus slowing down the observed dynamics and flattening the spectral ratios observed in HHS experiments.

\section{Conclusions}

We have formulated and tested a new accurate theoretical model for nuclear motion probed by high-harmonic spectroscopy. The model connects the observables of classical photoelectron spectroscopy to the intensity distribution in high-harmonic spectra and allows the inclusion of vibrational-state-dependent strong-field ionization rates. This enabled us to demonstrate experimentally and theoretically that the nuclear wave packet dynamics prepared by strong-field ionization differs quantitatively from a prediction based solely on Franck-Condon factors. The nuclear dynamics observed in HHS thus significantly differs from that prepared by single-photon ionization. We further showed that the relative populations of the vibronic eigenstates of the cation and consequently the launched nuclear dynamics can be controlled through the intensity of the laser field. Our model calculations shown in Fig. 2 further show that more complex nuclear dynamics than previously expected can be observed by HHS when the fundamental wavelength is extended to the mid-infrared, reaching electron transit times in excess of 4-5 fs. On these time scales, the quantization of the vibrational structure of the cation plays a role, although the vibrational periods themselves are much longer. Measurements accessing these time scales have recently become possible through the development of high-power few-cycle midinfrared laser sources [24], which have already been applied to generate high harmonics in the $\mathrm{keV}$ range [25]. Our results thus open new prospects for the investigation of nuclear dynamics by HHS by extending the wavelength range to the mid-infrared where the observation of vibronic quantum structure of nuclear motion is predicted.

Note added: Both the global shape and the local maximum of the ratio of autocorrelation functions shown in Fig. $2 \mathrm{f}$ have been confirmed in calculations based on the time-dependent Schrödingier equation [26]. These calculations further provide a time-dependent perspective of the origin of the local maximum.

\section{Acknowledgments}

We gratefully acknowledge funding from the Swiss National Science Foundation (PP00P2_128274) and ETH Zürich (ETH-33 10-3). We thank Alejandro Saenz and Stefan Haessler for fruitful discussions. 


\section{References}

[1] H. Niikura, F. Légaré, R. Hasbani, A. D. Bandrauk, M. Y. Ivanov, D. M. Villeneuve and P. B. Corkum, Nature 2002, 417, 917.

[2] H. Niikura, F. Légaré, R. Hasbani, M. Ivanov, D. M. Villeneuve and P. B. Corkum, Nature 2003, 421, 826.

[3] M. Lein, Phys. Rev. Lett. 2005, 94, 053004.

[4] S. Baker, J. S. Robinson, C. A. Haworth, H. Teng, R. A. Smith, C. C. Chirila, M. Lein, J. W. G. Tisch and J. P. Marangos, Science 2006, 312, 424 .

[5] S. Baker, J. S. Robinson, M. Lein, C. C. Chirila, R. Torres, H. C. Bandulet, D. Comtois, J. C. Kieffer, D. M. Villeneuve, J. W. G. Tisch and J. P. Marangos, Phys. Rev. Lett. 2008, 101, 053901.

[6] H. Mizutani, S. Minemoto, Y. Oguchi and H. Sakai, Journal of Physics B: Atomic, Molecular and Optical Physics 2011, 44, 081002.

[7] T. Kanai, E. J. Takahashi, Y. Nabekawa, and K. Midorikawa, New Journal of Physics 2008, 10, 025036.

[8] S. Haessler, B. Fabre, J. Higuet, J. Caillat, T. Ruchon, P. Breger, B. Carré, E. Constant, A. Maquet, E. Mével, P. Salières, R. Taïeb and Y. Mairesse, Phys. Rev. A 2009, 80, 011404.

[9] J. P. Farrell, S. Petretti, J. Förster, B. K. McFarland, L. S. Spector, Y. V. Vanne, P. Decleva, P. H. Bucksbaum, A. Saenz and M. Gühr, Phys. Rev. Lett. 2011, 107, 083001.

[10] A. D. Bandrauk, S. Chelkowski, S. Kawai and H. Lu, Phys. Rev. Lett. 2008, 101, 153901.

[11] A. D. Bandrauk, S. Chelkowski and H. Lu, Journal of Physics B: Atomic, Molecular and Optical Physics 2009, 42, 075602.

[12] S. Patchkovskii, Phys. Rev. Lett. 2009, 102, 253602.

[13] C. B. Madsen, M. Abu-samha and L. B. Madsen, Phys. Rev. A 2010, 81, 043413.

[14] A. Saenz, Journal of Physics B: Atomic, Molecular and Optical Physics 2000, 33, 4365.

[15] A. Becker, A. Bandrauk and S. Chin, Chemical Physics Letters 2001, 343, 345.

[16] T. K. Kjeldsen and L. B. Madsen, Phys. Rev. A 2005, 71, 023411. 
[17] X. Urbain, B. Fabre, E. M. Staicu-Casagrande, N. Ruettede, V. M. Andrianarijaona, J. Jureta, J. H. Posthumus, A. Saenz, E. Baldit and C. Cornaggia, Phys. Rev. Lett. 2004, 92, 163004.

[18] E. J. Heller, Accounts of Chemical Research 1981, 14, 368.

[19] E. Goll, G. Wunner and A. Saenz, Phys. Rev. Lett. 2006, 97, 103003.

[20] L. V. Keldysh, Sov. Phys. JETP 1965, 20, 1307.

[21] R. Locht, B. Leyh, K. Hottmann and H. Baumgärtel, Chemical Physics 1998, 233, 145.

[22] A. L. Stancik and E. B. Brauns, Vibrational Spectroscopy 2008, 47, 66.

[23] P. B. Corkum, Phys. Rev. Lett. 1993, 71, 1994.

[24] G. Andriukaitis, T. Balčiūnas, S. Ališauskas, A. Pugžlys, A. Baltuška, T. Popmintchev, M.-C. Chen, M. M. Murnane and H. C. Kapteyn, Opt. Lett. 2011, 36, 2755.

[25] T. Popmintchev, M.-C. Chen, D. Popmintchev, P. Arpin, S. Brown, S. Ališauskas, G. Andriukaitis, T. Balčiunas, O. D. Mücke, A. Pugzlys, A. Baltuška, B. Shim, S. E. Schrauth, A. Gaeta, C. Hernández-García, L. Plaja, A. Becker, A. Jaron-Becker, M. M. Murnane and H. C. Kapteyn, Science 2012, 336, 1287.

[26] J. Förster and A. Saenz, in preparation. 


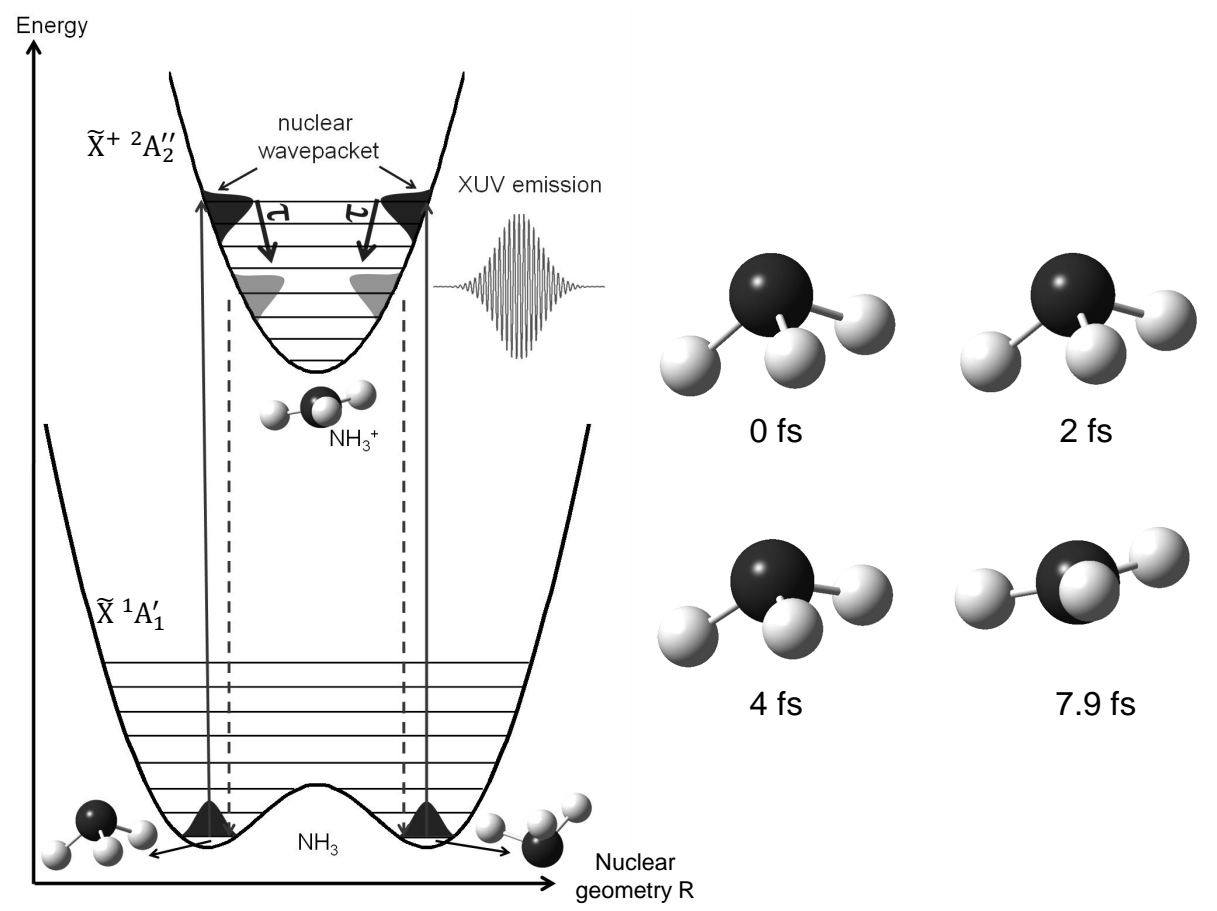

Figure 1: Left panel: Scheme for probing attosecond nuclear dynamics through HHS: strong-field ionization prepares a nuclear wave packet in the electronic ground state of the cation, the temporal evolution of which is probed by the recombining electron. Right panel: calculated structure of $\mathrm{NH}_{3}^{+}$at different time delays after ionization within a harmonic approximation of the potential energy surface of the cation. 

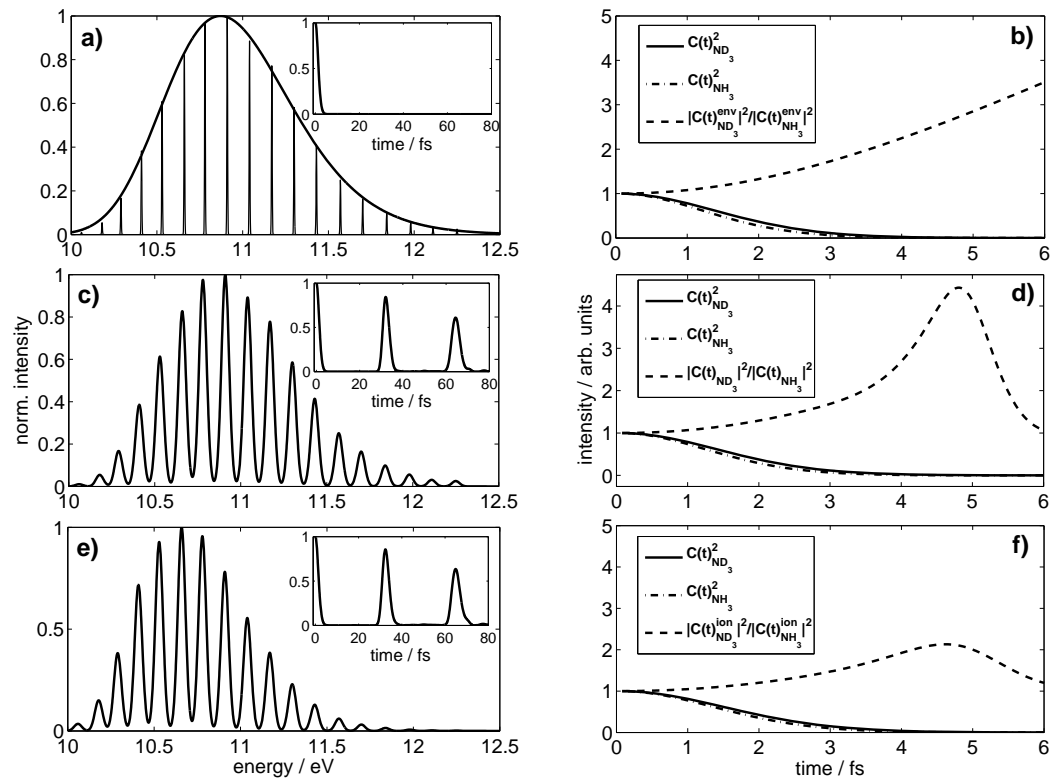

Figure 2: a) Photoelectron spectrum of the $\tilde{\mathrm{X}}^{2} \mathrm{~A}_{2}^{\prime \prime}$ state of $\mathrm{NH}_{3}^{+}$taken from Ref. [21] shown as a discrete stick spectrum and the envelope determined in a least-squares fitting procedure. The squared autocorrelation function obtained from the inverse Fourier transform of the spectral envelope is plotted in the inset. c) Experimentally observed photoelectron spectrum from Ref. [21] and the corresponding squared nuclear autocorrelation function shown as inset. e) Spectrum from c) weighted by the strong-field ionization rate $r_{\text {ion }}$ for an intensity of $5.0 \cdot 10^{13} \mathrm{~W} / \mathrm{cm}^{2}$ which is a realistic spectral representation of the launched nuclear wave packet. The squared autocorrelation function (inset) is calculated through an inverse Fourier transformation of the photoelectron spectrum weighted by the strong-field ionization amplitude $\left(\sqrt{r_{\text {ion }}}\right.$, see text). b) Ratio (red) of the squared autocorrelation function of $\mathrm{NH}_{3}$ (green line) and $\mathrm{ND}_{3}$ (blue line) for the band envelope shown in a). e) Analogous to b) for the discrete spectrum in c). f) Same as d) for the strong-field ionization weighted spectrum from e) and an equally processed spectrum of $\mathrm{ND}_{3}$. 


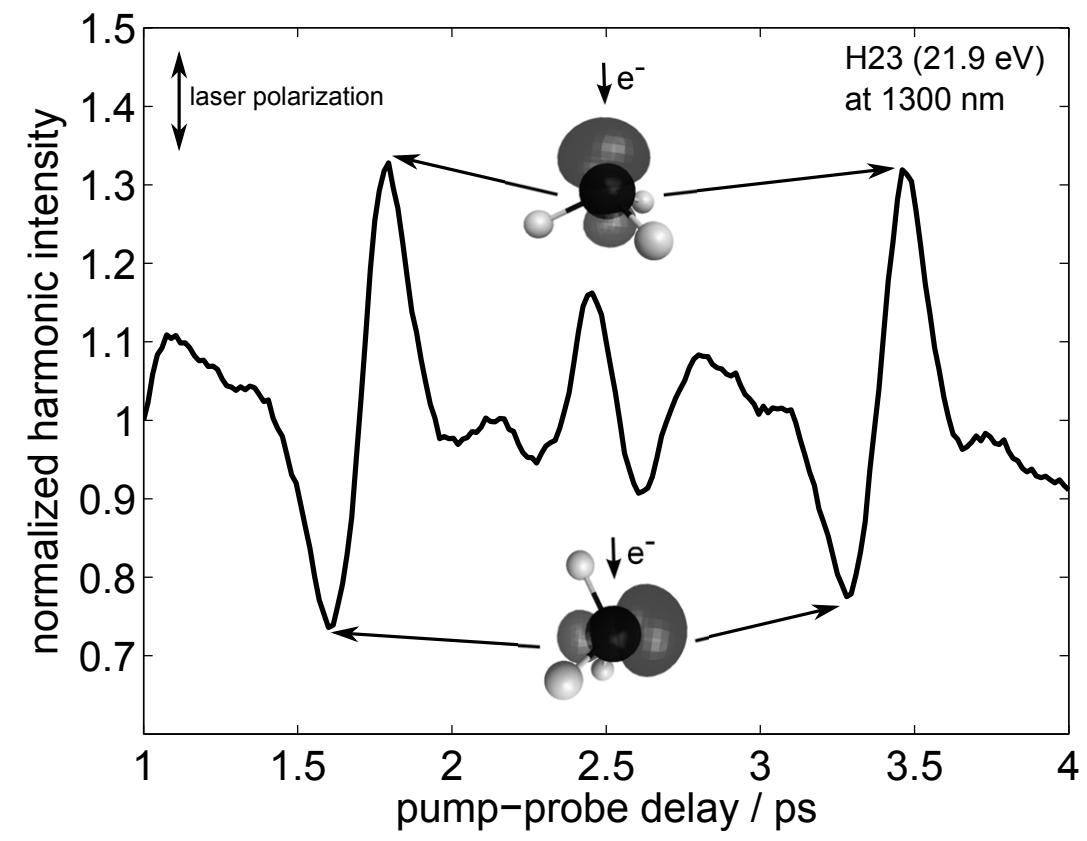

Figure 3: Rotational revivals of $\mathrm{NH}_{3}$ created through interaction of the molecules with a non-resonant $0.8 \mu \mathrm{m}$ laser pulse and probed by high-harmonic generation of a $1.3 \mu \mathrm{m}$ laser pulse. The figure shows the evolution of the intensity of H23 (corresponding to a photon energy of $21.9 \mathrm{eV}$ ) with the pump-probe delay. The polarization of the two laser pulses was parallel. The insets show the highest-occupied molecular orbital of the molecule as an isosurface representation with color-coded sign. 

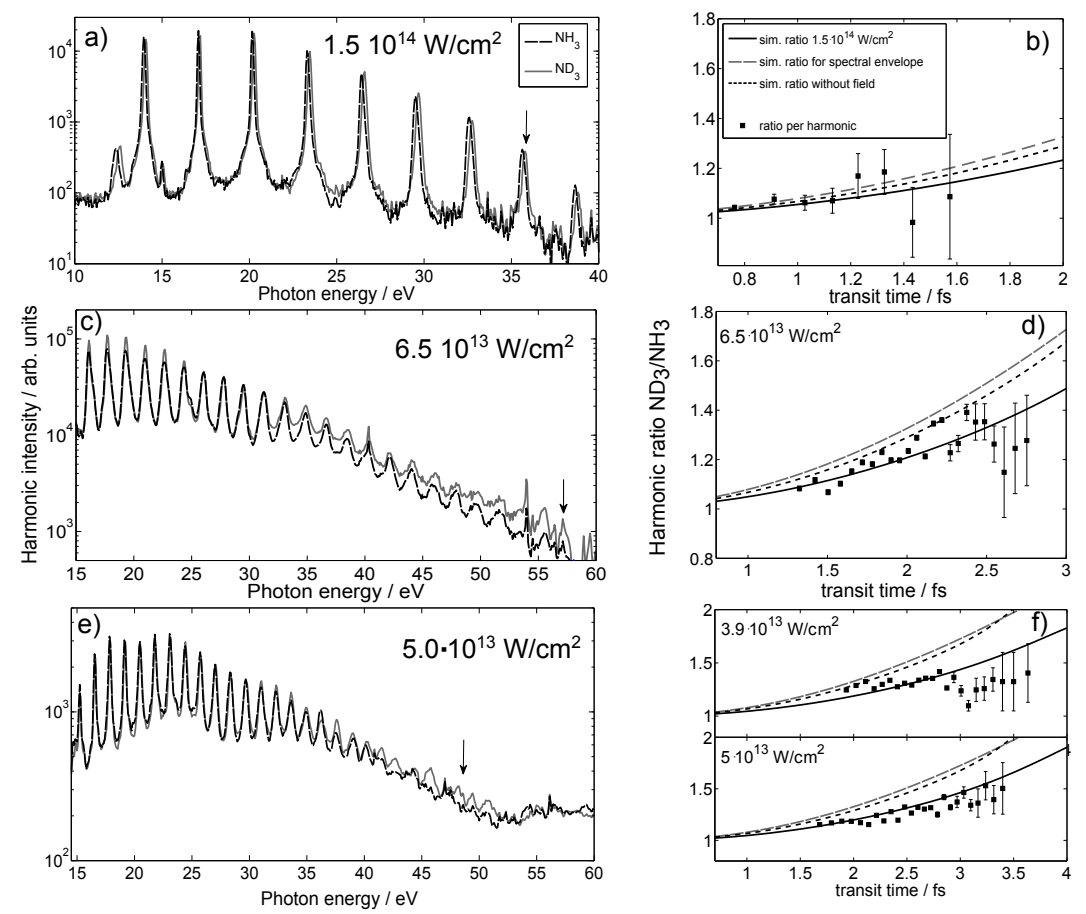

Figure 4: a) High-harmonic spectra of $\mathrm{NH}_{3}$ and $\mathrm{ND}_{3}$ recorded with laser pulses centered at $0.8 \mu \mathrm{m}$ and a peak intensity of $1.5 \cdot 10^{14} \mathrm{~W} / \mathrm{cm}^{2}$. The arrow indicates the highest photon energy taken into account in determining the spectral ratios. b) The ratio of the spectra from a) (squares) as a function of the electron transit time and predicted spectral ratios considering the spectral envelope only (dashdotted green line), the vibrationally-resolved photoelectron band (dashed red line) or the vibrationally-resolved band corrected by the strong-field ionization rate (full black line). The error bars were estimated from the signal-to-noise levels. c) Spectra recorded with laser pulses centered at $1.44 \mu \mathrm{m}$ and a peak intensity of $6.5 \cdot 10^{13} \mathrm{~W} / \mathrm{cm}^{2}$ and $\mathrm{d}$ ) the corresponding ratio and calculations. e) Spectra recorded with laser pulses centered at $1.8 \mu \mathrm{m}$ and a peak intensity of $5.0 \cdot 10^{13} \mathrm{~W} / \mathrm{cm}^{2}$. f) Ratios for two different peak intensities shown together with the corresponding theoretical predictions. 